\title{
Homoclinic Solutions of a Class of Nonperiodic Discrete Nonlinear Systems in Infinite Higher Dimensional Lattices
}

\author{
Genghong Lin ${ }^{1,2}$ and Zhan Zhou ${ }^{1,2}$ \\ ${ }^{1}$ School of Mathematics and Information Science, Guangzhou University, Guangzhou, Guangdong 510006, China \\ ${ }^{2}$ Key Laboratory of Mathematics and Interdisciplinary Sciences of Guangdong Higher Education Institutes, Guangzhou University, \\ Guangzhou, Guangdong 510006, China
}

Correspondence should be addressed to Zhan Zhou; zzhou0321@hotmail.com

Received 26 February 2014; Accepted 12 June 2014; Published 7 July 2014

Academic Editor: Chuangxia Huang

Copyright (C) 2014 G. Lin and Z. Zhou. This is an open access article distributed under the Creative Commons Attribution License, which permits unrestricted use, distribution, and reproduction in any medium, provided the original work is properly cited.

By using critical point theory, we obtain a new sufficient condition on the existence of homoclinic solutions of a class of nonperiodic discrete nonlinear systems in infinite lattices. The classical Ambrosetti-Rabinowitz superlinear condition is improved by a general superlinear one. Some results in the literature are improved.

\section{Introduction}

Assume that $m$ is a positive integer. Consider the following difference equation in infinite higher dimensional lattices:

$$
L u_{n}-\omega u_{n}=\sigma \gamma_{n} f_{n}\left(u_{n}\right), \quad n \in Z^{m},
$$

where $f_{n}(u)$ is continuous in $u, \sigma= \pm 1, n=\left(n_{1}, n_{2}, \ldots, n_{m}\right) \in$ $Z^{m}, \gamma=\left\{\gamma_{n}\right\}$ is a positive real valued sequence, $\omega \in R$, and $L$ is a Jacobi operator [1] given by

$$
\begin{aligned}
L u_{n}= & a_{1\left(n_{1}, n_{2}, \ldots, n_{m}\right)} u_{\left(n_{1}+1, n_{2}, \ldots, n_{m}\right)} \\
& +a_{1\left(n_{1}-1, n_{2}, \ldots, n_{m}\right)} u_{\left(n_{1}-1, n_{2}, \ldots, n_{m}\right)} \\
& +a_{2\left(n_{1}, n_{2}, \ldots, n_{m}\right)} u_{\left(n_{1}, n_{2}+1, \ldots, n_{m}\right)} \\
& +a_{2\left(n_{1}, n_{2}-1, \ldots, n_{m}\right)} u_{\left(n_{1}, n_{2}-1, \ldots, n_{m}\right)} \\
& +\cdots+a_{m\left(n_{1}, n_{2}, \ldots, n_{m}\right)} u_{\left(n_{1}, n_{2}, \ldots, n_{m}+1\right)} \\
& +a_{m\left(n_{1}, n_{2}, \ldots, n_{m}-1\right)} u_{\left(n_{1}, n_{2}, \ldots, n_{m}-1\right)} \\
& +b_{\left(n_{1}, n_{2}, \ldots, n_{m}\right)} u_{\left(n_{1}, n_{2}, \ldots, n_{m}\right)}
\end{aligned}
$$

here, $\left\{a_{i n}\right\}(i=1,2, \ldots, m)$ and $\left\{b_{n}\right\}$ are real valued bounded sequences.
Assume that $f_{n}(0)=0$ for $n \in Z^{m}$; then $\left\{u_{n}\right\}=\{0\}$ is a solution of (1), which is called the trivial solution. As usual, we say that $u=\left\{u_{n}\right\}$, a solution of (1), is homoclinic (to 0 ) if

$$
\lim _{|n| \rightarrow \infty} u_{n}=0
$$

where $|n|=\left|n_{1}\right|+\left|n_{2}\right|+\cdots+\left|n_{m}\right|$ is the length of multiindex $n$. In addition, if $\left\{u_{n}\right\} \neq\{0\}$, then $u$ is called a nontrivial homoclinic solution. We are interested in the existence of the nontrivial homoclinic solutions for (1). This problem appears when we seek the discrete solitons of nonperiodic discrete nonlinear Schrödinger (DNLS) equation

$$
i \dot{\psi}_{n}=-\Delta \psi_{n}-\sigma \gamma_{n} f_{n}\left(\psi_{n}\right), \quad n \in Z^{m},
$$

where $\sigma= \pm 1$ and

$$
\begin{aligned}
\Delta \psi_{n}= & \psi_{\left(n_{1}+1, n_{2}, \ldots, n_{m}\right)} \\
& +\psi_{\left(n_{1}-1, n_{2}, \ldots, n_{m}\right)}+\psi_{\left(n_{1}, n_{2}+1, \ldots, n_{m}\right)} \\
& +\psi_{\left(n_{1}, n_{2}-1, \ldots, n_{m}\right)}+\cdots+\psi_{\left(n_{1}, n_{2}, \ldots, n_{m}+1\right)} \\
& +\psi_{\left(n_{1}, n_{2}, \ldots, n_{m}-1\right)}-2 m \psi_{\left(n_{1}, n_{2}, \ldots, n_{m}\right)}
\end{aligned}
$$

is the discrete Laplacian in $m$ spatial dimension. Typical representatives of power nonlinearities are

$$
f_{n}(u)=l_{n}|u|^{p} u, \quad l_{n}, p>0 .
$$


Primarily, we are interested in spatially localized, or solitary, standing waves. Such waves are often called breathers or gap solitons. The origin of the last name is that typically such solutions do exist for frequencies in gaps of linear spectrum. Considering (4), we suppose that the nonlinearity is gauge invariant; that is,

$$
f_{n}\left(e^{i \theta} u\right)=e^{i \theta} f_{n}(u), \quad \theta \in R,
$$

and, in addition, $f_{n}(u) \geq 0$ for $u \geq 0$ for $n \in Z^{m}$.

Making use of the standing wave ansatz,

$$
\begin{gathered}
\psi_{n}=u_{n} e^{-i \omega t}, \\
\lim _{|n| \rightarrow \infty} \psi_{n}=0
\end{gathered}
$$

where $\left\{u_{n}\right\}$ is a real valued sequence and $\omega \in R$ is the temporal frequency. Then (4) becomes

$$
-\Delta u_{n}-\omega u_{n}=\sigma \gamma_{n} f_{n}\left(u_{n}\right), \quad n \in Z^{m},
$$

and (3) holds. This is an equation of the form (1) with $a_{i n}=$ $-1(i=1,2, \ldots, m)$ and $b_{n}=2 m$.

When $f_{n}(u)$ has the form of (6), the homoclinic solutions of (9) were obtained by Karachalios in [2] by assuming that $\gamma \in l^{\rho}, \rho=(q-1) /(q-2)$, for some $q>2$. We note that $\gamma \in l^{\rho}$ implies that $\lim _{|n| \rightarrow \infty} \gamma_{n}=0$. Moreover, (6) satisfies the classical Ambrosetti-Rabinowitz superlinear condition [3], and $f_{n}(u) /|u|$ is nondecreasing with respect to $|u|$, both of which played important roles in the existence of homoclinic solutions of (1.7) in [2].

The aim of this paper is to improve both the monotone condition of $f_{n}(u) /|u|$ and the classical AmbrosettiRabinowitz superlinear condition by general ones; see Remarks 8 and 9 for details. Moreover, in this paper, we only need $\lim _{|n| \rightarrow \infty} \gamma_{n}=0$. Particularly, our results improved the results in [2]; see Remarks 3 and 7 for details.

In the past years, there has been large growth in the study of DNLS equation, which is a nonlinear lattice system that appears in many areas of physics. Discrete solitons which exist in DNLS systems, that is, solitary waves and localized structures in spatially discrete media, are also of particular interest in their own right. Among these, one can mention photorefractive media [4], biomolecular chains [5], and Bose-Einstein condensates [6]. The experimental observations of discrete solitons in nonlinear lattice systems have been reported [7-11]. To mention that, many authors have studied the existence of discrete solitons of the DNLS equations [12-17]. The fruitful methods include centre manifold reduction [16], variational methods $[12,14]$, the principle of anticontinuity $[13,17]$, and the Nehari manifold approach [18]. However, most of the existing literature is devoted to the DNLS equations with constant coefficients or periodic coefficients. Results on such DNLS equations have been summarized in [19-23]. And we also want to mention that, in recent years, the existence of homoclinic solutions for difference equations has been studied by many authors, and we refer to [24-36].

Since the operator $L$ is bounded and self-adjoint in the space $l^{2}$ (defined in Section 2), we consider (1) as a nonlinear equation in $l^{2}$ with (3) being satisfied automatically. The spectrum $\sigma(L)$ of $L$ is closed. Thus, the complement $R \backslash \sigma(L)$ consists of a finite number of open intervals called spectral gaps and two of them are semi-infinite which are denoted by $(-\infty, \beta)$ and $(\alpha, \infty)$, respectively. In this paper, we consider the homoclinic solutions of (1) in $l^{2}$ for the case where $\omega \epsilon$ $(-\infty, \beta)$ and $\sigma=1$. The case where $\omega \in(\alpha, \infty)$ and $\sigma=-1$ is omitted, since, in this case, we can replace $L$ by $-L$.

The main idea in this paper is as follows. First, we assume that $\left\{\gamma_{n}\right\}$ converges to zero at infinity; that is, $\lim _{|n| \rightarrow \infty} \gamma_{n}=0$. After that, we prove a compact inclusion between ordinary sequence spaces $l^{2}$ and weighted sequence spaces $l_{\gamma}^{2}$ (defined in Section 2), in order to come over lack of compactness for the so-called $(C)_{c}$ condition (defined in Section 2). Finally, by making use of the Mountain Pass Lemma [37], we prove the existence of homoclinic solutions of (1) in $l^{2}$.

\section{Preliminaries}

In this section, we first establish the variational setting associated with (1). Let

$$
\begin{array}{r}
l^{p}=l^{p}\left(Z^{m}\right)=\left\{u=\left\{u_{n}\right\}_{n \in Z^{m}}: \forall n \in Z^{m}, u_{n} \in R,\right. \\
\left.\|u\|_{l^{p}}=\left(\sum_{n \in Z^{m}}\left|u_{n}\right|^{p}\right)^{1 / p}<\infty\right\} .
\end{array}
$$

Then the following embedding between $l^{p}$ spaces holds:

$$
l^{q} \subset l^{p}, \quad\|u\|_{l^{p}} \leq\|u\|_{l^{q}}, \quad 1 \leq q \leq p \leq \infty .
$$

For $p=2$, we get the usual Hilbert space of square-summable sequences, with the real scalar product

$$
(u, v)_{l^{2}}=\sum_{n \in Z^{m}} u_{n} v_{n}, \quad u, v \in l^{2} .
$$

For a positive real valued bounded sequence $\gamma=$ $\left\{\gamma_{n}: 0<\gamma_{n} \leq \bar{\gamma}<\infty\right\}_{n \in Z^{m}}$, we define the weighted sequence spaces $l_{\gamma}^{2}$ :

$$
\begin{gathered}
l_{\gamma}^{2}=\left\{u=\left\{u_{n}\right\}_{n \in Z^{m}}: \forall n \in Z^{m}, u_{n} \in R,\right. \\
\left.\|u\|_{l_{\gamma}^{2}}=\left(\sum_{n \in Z^{m}} \gamma_{n}\left|u_{n}\right|^{2}\right)^{1 / 2}<\infty\right\} .
\end{gathered}
$$

It is not hard to see that $l_{\gamma}^{2}$ is a Hilbert space, with the scalar product

$$
(u, v)_{l_{\gamma}^{2}}=\sum_{n \in Z^{m}} \gamma_{n} u_{n} v_{n}, \quad u, v \in l_{\gamma}^{2}
$$

For a certain class of weight $\gamma$, we have the following lemmas, which will play a crucial role in our analysis. 
Lemma 1. Let $\kappa=\left\{\kappa_{n}:\left|\kappa_{n}\right| \leq \bar{\kappa}<\infty\right\}_{n \in Z^{m}}$ be a multiplication operator from $l_{\gamma}^{2}$ to $l_{\gamma}^{2}$ defined by $\kappa u=\left\{\kappa_{n} u_{n}\right\}_{n \in Z^{m}}$. If $\lim _{|n| \rightarrow \infty} \kappa_{n}=0$, then the operator $\kappa$ is compact.

Proof of Lemma 1. Let

$$
\Lambda=\left\{\kappa u:\|u\|_{l_{\gamma}^{2}} \leq 1\right\} .
$$

We only need to prove that $\Lambda$ is precompact in $l_{\gamma}^{2}$. By assumption, for any $\varepsilon>0$, there exists $N>0$ such that $\left|\kappa_{n}\right| \leq \varepsilon$ for any $|n|>N$. Define a cutting sequence $\chi=\left\{\chi_{n}\right\}$ by

$$
\chi_{n}= \begin{cases}1, & |n| \leq N \\ 0, & |n|>N .\end{cases}
$$

Denote by $\chi^{c}=1-\chi$ the anticutting sequence. Then for any $\kappa u \in \Lambda$

$$
\begin{aligned}
\left\|\chi^{c} \kappa u\right\|_{l_{\gamma}^{2}}^{2} & =\sum_{|n|>N} \gamma_{n}\left|\kappa_{n} u_{n}\right|^{2} \leq \varepsilon^{2}, \\
\|\chi \kappa u\|_{l_{\gamma}^{2}}^{2} & =\sum_{|n| \leq N} \gamma_{n}\left|\kappa_{n} u_{n}\right|^{2} \leq \bar{\kappa}^{2} .
\end{aligned}
$$

For arbitrary $\varepsilon>0$ and $\Lambda_{\varepsilon}=\left\{\chi \mathcal{K} u:\|u\|_{l_{\gamma}^{2}} \leq 1\right\}$ finitedimensional and bounded, we know that $\Lambda$ is precompact. The proof is complete.

Lemma 2. One assumes positive sequence of real numbers $\gamma$ with $\lim _{|n| \rightarrow \infty} \gamma_{n}=0$. Then $l^{2} \hookrightarrow l_{\gamma}^{2}$ with compact inclusion.

Proof of Lemma 2. Note that $\|u\|_{l_{\nu}^{2}} \leq \sqrt{\bar{\gamma}}\|u\|_{l^{2}}$ for any $u \in l^{2}$ and $\gamma^{1 / 2}$ is compact. Thus, $l^{2} \hookrightarrow l_{\gamma}^{2}$ with compact inclusion by Lemma 1 . The proof is complete.

Remark 3. Karachalios [2] proved $l^{2} \hookrightarrow l_{\gamma}^{2}$ with compact inclusion assuming that $\gamma \in l^{\rho}, \rho=(q-1) /(q-2)$, for some $q>2$. Note that $\gamma \in l^{\rho}$ implies that $\lim _{|n| \rightarrow \infty} \gamma_{n}=0$. Thus, we find that Lemma 2 improves Lemma 2.1 in [2].

On the Hilbert space $l^{2}$, we consider the functional

$$
J(u)=\frac{1}{2}((L-\omega) u, u)_{l^{2}}-\sigma \sum_{n \in Z^{m}} \gamma_{n} F_{n}\left(u_{n}\right),
$$

where

$$
F_{n}(u)=\int_{0}^{u} f_{n}(s) d s
$$

is the primitive function of $f_{n}(u)$. Then $J \in C^{1}\left(l^{2}, R\right)$ and

$$
\begin{aligned}
\left\langle J^{\prime}(u), v\right\rangle= & ((L-\omega) u, v)_{l^{2}} \\
& -\sigma \sum_{n \in Z^{m}} \gamma_{n} f_{n}\left(u_{n}\right) v_{n}, \quad u, v \in l^{2} .
\end{aligned}
$$

Equation (20) implies that (1) is the corresponding EulerLagrange equation for $J$. Therefore, we have reduced the problem of finding a nontrivial homoclinic solution of (1) to that of seeking a nonzero critical point of the functional $J$ on $l^{2}$.

Let $\delta$ be the distance from $\omega$ to the spectrum $\sigma(L)$; that is,

$$
\delta=\beta-\omega
$$

Then, we have

$$
((L-\omega) u, u) \geq \delta\|u\|_{l^{2}}^{2}, \quad u \in l^{2} .
$$

We also consider a norm in $l^{2}$ defined by

$$
\|u\|_{l_{\omega}^{2}}=[((L-\omega) u, u)]^{1 / 2}, \quad u \in l^{2} .
$$

Since

$$
\delta\|u\|_{l^{2}}^{2} \leq\|u\|_{l_{\omega}^{2}}^{2} \leq\|L-\omega\|\|u\|_{l^{2}}^{2}, \quad u \in l^{2},
$$

norm (23) is an equivalent norm with the usual one of $l^{2}$.

In order to obtain the existence of critical points of $J$ on $l^{2}$, we cite some basic notations and some known results from critical point theory.

Let $H$ be a Hilbert space and $C^{1}(H, R)$ denote the set of functionals that are Fréchet differentiable and their Fréchet derivatives are continuous on $H$.

Let $J \in C^{1}(H, R)$. A sequence $\left\{u_{j}\right\} \subset H$ is called a $(C)_{c}$ sequence for $J$ if $J\left(u_{j}\right) \rightarrow c$ for some $c \in R$ and $\left(1+\left\|u_{j}\right\|\right)\left\|J^{\prime}\left(u_{j}\right)\right\|$ as $j \rightarrow \infty$. We say $J$ satisfies the $(C)_{c}$ condition if any $(C)_{c}$ sequence for $J$ possesses a convergent subsequence.

Let $B_{r}$ be the open ball in $H$ with radius $r$ and center 0 , and let $\partial B_{r}$ denote its boundary. The following lemma is taken from [37].

Lemma 4 (Mountain Pass Lemma). If $J \in C^{1}(H, R)$ and satisfies the following conditions: there exist $e \in H \backslash\{0\}$ and $r \in(0,\|e\|)$ such that $\max \{J(0), J(e)\}<\inf _{\|u\|=r} J(u)$, then there exists a $(C)_{c}$ sequence $\left\{u_{n}\right\}$ for the mountain pass level $c$ which is defined by

$$
c=\inf _{h \in \Gamma s \in[0,1]} \max _{(h)} J(h)
$$

where

$$
\Gamma=\{h \in C([0,1], H): h(0)=0, h(1)=e\} .
$$

\section{Main Results}

In this section, we will establish some sufficient conditions on the existence of nontrivial solutions of (1) in $l^{2}$.

Theorem 5. Assume that $\sigma=1, \omega \in(-\infty, \beta)$, and the following conditions hold.

(H1) $f_{n}(u)$ is continuous in $u, f_{n}(u)=o(u)$ as $u \rightarrow 0$ uniformly for $n \in Z^{m}$.

(H2) There exist $b>0, p>2$ such that

$$
\left|f_{n}(u)\right| \leq b\left(1+|u|^{p-1}\right)
$$

uniformly for $n \in Z^{m}$ and $u \in R$. 
(H3) There exists some $\theta \geq 1$ such that $\theta G_{n}(u) \geq G_{n}(t u)$, for $n \in Z^{m}, u \in R$, and $t \in[0,1]$, where $G_{n}(u)=$ $(1 / 2) f_{n}(u) u-F_{n}(u)$.

(H4) $F_{n}(u) \geq 0$ for $u \in R$, and $\lim _{|u| \rightarrow \infty}\left(F_{n}(u) / u^{2}\right)=\infty$, uniformly for $n \in Z^{m}$.

(H5) Positive real valued sequence $\gamma=\left\{\gamma_{n}\right\}_{n \in Z^{m}}$ with $\lim _{|n| \rightarrow \infty} \gamma_{n}=0$.

Then (1) has at least a nontrivial solution $u$ in $l^{2}$ and the solution decays exponentially at infinity. That is, there exist two positive constants $C$ and $\tau$ such that

$$
\left|u_{n}\right| \leq C e^{-\tau|n|}, \quad n \in Z^{m} .
$$

Theorem 5 gives some sufficient conditions on the existence of nontrivial solutions of (1) in $l^{2}$. However, (1) may have no nontrivial solutions in $l^{2}$. In fact, we have the following proposition.

Proposition 6. Assume that $\sigma=-1, \omega \leq \beta$, and $\gamma_{n} f_{n}(u) u>0$ when $u \neq 0$ for all $n \in Z^{m}$. Then (1) has no nontrivial solutions in $l^{2}$.

Proof of Proposition 6. By way of contradiction, we assume that (1) has a nontrivial solution $u=\left\{u_{n}\right\} \in l^{2}$. Then $u$ is a nonzero critical point of $J$, and

$$
\begin{aligned}
\left\langle J^{\prime}(u), u\right\rangle & =((L-\omega) u, u)_{l^{2}}-\sigma \sum_{n \in Z^{m}} \gamma_{n} f_{n}\left(u_{n}\right) u_{n} \\
& \geq \sum_{n \in Z^{m}} \gamma_{n} f_{n}\left(u_{n}\right) u_{n}>0
\end{aligned}
$$

This is a contradiction as $\left\langle J^{\prime}(u), u\right\rangle=0$, so the conclusion holds.

Remark 7. It is easy to see that the function $f_{n}$ defined by

$$
f_{n}(u)=|u|^{2 \lambda} u,
$$

where $\lambda>0$ and $\gamma \in l^{\rho}, \rho=(q-1) /(q-2)$, for some $q>2$, satisfies all conditions in Theorem 5 . This case was studied by [2], and we find that Theorem 5 improves Theorem 2.3 in [2].

Remark 8. We will introduce another condition $(\bar{H} 3)$ : $f_{n}(u) /|u|$ is nondecreasing with respect to $|u|$. We want to point out that condition $(H 3)$ is equivalent to $(\bar{H} 3)$ when $\theta=1$ and (H3) gives "better monotony" when $\theta>1$, since $(\bar{H} 3)$ implies $(H 3)$ (see [38]). Moreover, we can find that $f_{n}(u)=5 u \ln \left(1+u^{2}\right)+9 \sin u$ satisfies (H3) but not $(\bar{H} 3)$ for some $\theta \geq 100$.

Remark 9. As we know, the condition

$(\bar{H} 4) f(u) u>q F(u)>0$ for some $q>2$ and $u \neq 0$

is often called Ambrosetti-Rabinowitz superlinear condition [3]. Clearly, $(\bar{H} 4)$ implies (H4). Actually, $(H 4)$ is more general than $(\bar{H} 4)$. Let $\left\{a_{n}\right\}$ be a positive sequence, and $f_{n}(u)=$ $a_{n} u \ln (1+|u|)$. Then $f_{n}$ satisfies (H4). However, $f_{n}$ does not satisfy $(\bar{H} 4)$.
The proof of Theorem 5 is based on a direct application of the following lemmas. The key points read as follows.

Lemma 10. Assume that the conditions of Theorem 5 hold; then one has the following.

$\left(J_{1}\right)$ There exist two constants $a>0$ and $\rho>0$ such that $\left.J\right|_{\partial B_{\rho}} \geq a$.

$\left(J_{2}\right)$ There exists an $e \in l^{2}$ such that $J(t e) \rightarrow-\infty$ as $|t| \rightarrow$ $\infty$.

Proof of Lemma 10. Let $\bar{\gamma}=\max _{n \in Z^{m}}\left\{\gamma_{n}\right\}$ and $\epsilon=\delta / 2 \bar{\gamma}$. By $(H 1)$ and $(H 2)$, there exists $c_{1}>0$, such that

$$
\left|f_{n}(u)\right| \leq \epsilon|u|+c_{1}|u|^{p-1}
$$

for all $n \in Z^{m}$ and $u \in R$, and (31) implies that

$$
\left|F_{n}(u)\right| \leq \frac{\epsilon}{2}|u|^{2}+\frac{c_{1}}{p}|u|^{p} .
$$

By (32) and the Hölder inequality, we have

$$
\begin{aligned}
J(u) & =\frac{1}{2}((L-\omega) u, u)_{l^{2}}-\sum_{n \in Z^{m}} \gamma_{n} F_{n}\left(u_{n}\right) \\
& \geq \frac{\delta}{2}\|u\|_{l^{2}}^{2}-\bar{\gamma} \sum_{n \in Z^{m}}\left(\frac{\epsilon}{2}|u|^{2} \frac{c_{1}}{p}|u|^{p}\right) \\
& \geq \frac{\delta}{4}\|u\|_{l^{2}}^{2}-\frac{c_{1} \bar{\gamma}}{p}\|u\|_{l^{2}}^{p} .
\end{aligned}
$$

Since $p>2$, we have

$$
J(u) \geq \frac{\delta \rho^{2}}{8}=a>0 \text { for }\|u\|_{l^{2}}=\rho,
$$

where $\rho=\left(\delta p / 8 c_{1} \bar{\gamma}\right)^{1 /(p-2)}$.

Let $e=\left\{e_{n}\right\} \in l^{2}$ be the eigenvector of $L$ corresponding to the eigenvalue $\beta$; that is to say, $L e=\beta e$. There exists $N>0$, such that

$$
\sum_{|n| \leq N} e_{n}^{2} \geq \frac{1}{2}\|e\|_{l^{2}}^{2}
$$

Let

$$
A^{*}=\left\{n \in Z^{m}:|n| \leq N, e_{n} \neq 0\right\} .
$$

By $(H 4)$, for any $M>0$, there exists $\eta=\eta(M)>0$ such that

$$
F_{n}(u) \geq M|u|^{2} \quad \text { for } n \in Z^{m},|u| \geq \eta \text {. }
$$

Taking $t$ large enough, such that $t e_{n}>\eta$ for all $n \in A^{*}$, then, combining (35), (36), and (37), we have

$$
\begin{aligned}
J(t e) & =\frac{1}{2}((L-\omega) t e, t e)_{l^{2}}-\sum_{n \in Z^{m}} \gamma_{n} F_{n}\left(t e_{n}\right) \\
& \leq \frac{\delta}{2} t^{2}\|e\|_{l^{2}}^{2}-\sum_{n \in A^{*}} \gamma_{n} F_{n}\left(t e_{n}\right) \\
& \leq \frac{\delta}{2} t^{2}\|e\|_{l^{2}}^{2}-\underline{\gamma} M t^{2} \sum_{n \in A^{*}} e_{n}^{2} \\
& \leq \frac{1}{2}(\delta-\underline{\gamma} M) t^{2}\|e\|_{l^{2}}^{2},
\end{aligned}
$$


where $\underline{\gamma}=\min _{n \in A^{*}}\left\{\gamma_{n}\right\}>0$. Letting $M$ be large enough, such that $\delta \leq \gamma M$, we obtain that $J(t e) \rightarrow-\infty$ as $|t| \rightarrow \infty$. The proof is complete.

Lemma 11. Assume that the conditions of Theorem 5 hold; then the functional $J$ satisfies the $(C)_{c}$ condition for any given $c \in R$.

Proof of Lemma 11. Let $\left\{u^{(k)}\right\} \subset l^{2}$ be a $(C)_{c}$ sequence of $J$; that is,

$$
\begin{aligned}
J\left(u^{(k)}\right) & \longrightarrow c, \\
\left(1+\left\|u^{(k)}\right\|_{l^{2}}\right)\left\|J^{\prime}\left(u^{(k)}\right)\right\|_{l^{2}} & \longrightarrow 0, \\
\text { as } k & \longrightarrow \infty .
\end{aligned}
$$

First, we prove that $\left\{u^{(k)}\right\}$ is bounded in $l^{2}$. By way of contradiction, assume that $\left\|u^{(k)}\right\|_{l^{2}} \rightarrow \infty$ as $k \rightarrow \infty$. Set $\xi^{(k)}=u^{(k)} /\left\|u^{(k)}\right\|_{l^{2}}$. Up to a sequence, we have

$$
\begin{array}{ll}
\xi^{(k)}-\xi, & \text { in } l^{2}, \\
\xi^{(k)} \longrightarrow \xi, & \text { in } l_{\gamma}^{2} .
\end{array}
$$

Case $1(\xi \neq 0)$. By $J\left(u^{(k)}\right)=c+o(1)$, where $o(1) \rightarrow 0$ as $k \rightarrow 0$, we have

$$
\begin{aligned}
& \sum_{n \in Z^{m}} \gamma_{n} \frac{F_{n}\left(u_{n}^{(k)}\right)}{\left\|u^{(k)}\right\|_{l^{2}}^{2}}=\frac{1}{2} \frac{\left((L-\omega) u^{(k)}, u^{(k)}\right)}{\left\|u^{(k)}\right\|_{l^{2}}^{2}}-\frac{c+o(1)}{\left\|u^{(k)}\right\|_{l^{2}}^{2}} \\
& \leq \frac{\|L-\omega\|}{2}-\frac{c+o(1)}{\left\|u^{(k)}\right\|_{l^{2}}^{2}}<\infty .
\end{aligned}
$$

Let $B^{*}=\left\{n \in Z^{m}: \xi_{n} \neq 0\right\}$. Obviously, $B^{*}$ is nonempty. Then, for some $n_{0} \in B^{*}$, it follows from (41) that

$$
u_{n_{0}}^{(k)}=\xi_{n_{0}}^{(k)}\left\|u^{(k)}\right\|_{l^{2}} \longrightarrow \infty, \quad \text { as } k \longrightarrow \infty .
$$

Combining (H4) and $\gamma_{n_{0}}>0$, we have

$$
\gamma_{n_{0}} \frac{F_{n_{0}}\left(u_{n_{0}}^{(k)}\right)}{\left\|u^{(k)}\right\|_{l^{2}}^{2}}=\gamma_{n_{0}} \frac{F_{n_{0}}\left(u_{n_{0}}^{(k)}\right)}{\left|u_{n_{0}}^{(k)}\right|^{2}}\left|\xi_{n_{0}}^{(k)}\right|^{2} \longrightarrow \infty, \quad \text { as } k \longrightarrow \infty \text {. }
$$

However,

$$
\begin{aligned}
\sum_{n \in Z^{m}} \gamma_{n} \frac{F_{n}\left(u_{n}^{(k)}\right)}{\left\|u^{(k)}\right\|_{l^{2}}^{2}} & =\sum_{n \neq n_{0}} \gamma_{n} \frac{F_{n}\left(u_{n}^{(k)}\right)}{\left\|u^{(k)}\right\|_{l^{2}}^{2}}+\gamma_{n_{0}} \frac{F_{n_{0}}\left(u_{n_{0}}^{(k)}\right)}{\left\|u^{(k)}\right\|_{l^{2}}^{2}} \\
& \geq \gamma_{n_{0}} \frac{F_{n_{0}}\left(u_{n_{0}}^{(k)}\right)}{\left\|u^{(k)}\right\|_{l^{2}}^{2}} \longrightarrow \infty,
\end{aligned}
$$

as $k \rightarrow \infty$. This contradicts (42).

Case $2(\xi=0)$. Let

$$
J\left(t_{k} u^{(k)}\right)=\max _{t \in[0,1]} J\left(t u^{(k)}\right) .
$$

For any given $M>\max \{4, \theta c / 2 \delta\}$, let $k$ be large enough such that $\left\|u^{(k)}\right\|_{l^{2}}>M$ and $\bar{\xi}^{(k)}=2 M^{1 / 2} \xi^{(k)}$. Combining (32), (41), and $\xi=0$, it is easy to see that

$$
\begin{aligned}
& \sum_{n \in Z^{m}} \gamma_{n} F_{n}\left(\bar{\xi}_{n}^{(k)}\right) \\
& \leq \frac{\epsilon}{2}\left\|\bar{\xi}^{(k)}\right\|_{l_{\gamma}^{2}}^{2}+\frac{c_{1} \bar{\gamma}^{1 / 2}}{p}\left\|\bar{\xi}^{(k)}\right\|_{l^{2(p-1)}}^{p-1}\left\|\bar{\xi}^{(k)}\right\|_{l_{\gamma}^{2}} \longrightarrow 0, \\
& \text { as } k \longrightarrow \infty .
\end{aligned}
$$

Thus, for $k$ large enough, we have

$$
\begin{aligned}
J\left(t_{k} u^{(k)}\right) & \geq J\left(\bar{\xi}^{(k)}\right) \\
& \geq \frac{\delta}{2}\left\|\bar{\xi}^{(k)}\right\|_{l^{2}}^{2}-\sum_{n \in Z^{m}} \gamma_{n} F_{n}\left(\bar{\xi}_{n}^{(k)}\right) \\
& \geq 2 \delta M-\sum_{n \in Z^{m}} \gamma_{n} F_{n}\left(\bar{\xi}_{n}^{(k)}\right) .
\end{aligned}
$$

By (47), (48), and $M>c / 2 \delta$, we have

$$
\lim _{k \rightarrow \infty} J\left(t_{k} u^{(k)}\right) \geq 2 \delta M>\theta c .
$$

Noting that $J(0)=0$ and $J\left(u^{(k)}\right) \rightarrow c$, as $k \rightarrow \infty$, then $0<t_{k}<1$ when $k$ is big enough. Thus, $\left\langle J^{\prime}\left(t_{k} u^{(k)}\right), t_{k} u^{(k)}\right\rangle=0$. In view of $(H 3)$, it follows that

$$
\begin{aligned}
J\left(t_{k} u^{(k)}\right) & =J\left(t_{k} u^{(k)}\right)-\frac{1}{2}\left\langle J^{\prime}\left(t_{k} u^{(k)}\right), t_{k} u^{(k)}\right\rangle \\
& =\sum_{n \in Z^{m}} \gamma_{n}\left(\frac{1}{2} f_{n}\left(t_{k} u_{n}^{(k)}\right) t_{k} u_{n}^{(k)}-F_{n}\left(t_{k} u_{n}^{(k)}\right)\right) \\
& \leq \theta \sum_{n \in Z^{m}} \gamma_{n}\left(\frac{1}{2} f_{n}\left(u_{n}^{(k)}\right) u_{n}^{(k)}-F_{n}\left(u_{n}^{(k)}\right)\right) \\
& =\theta\left(J\left(u^{(k)}\right)-\frac{1}{2}\left\langle J^{\prime}\left(u^{(k)}\right), u^{(k)}\right\rangle\right) .
\end{aligned}
$$

By (50), we have

$$
\lim _{k \rightarrow \infty} J\left(t_{k} u^{(k)}\right) \leq \theta c
$$

This contradicts (49), so $\left\{u^{(k)}\right\}$ is bounded in $l^{2}$.

Second, we show that there exists a convergent subsequence of $\left\{u^{(k)}\right\}$. In fact, there exists a subsequence, still denoted by the same notation, such that

$$
u^{(k)}-u, \quad \text { in } l^{2} .
$$

By Lemma 2, we have

$$
u^{(k)} \longrightarrow u, \quad \text { in } l_{\gamma}^{2}
$$


By direct calculation, we obtain

$$
\begin{aligned}
\| u^{(k)}- & u \|_{l^{2}}^{2} \\
= & \left\langle J^{\prime}\left(u^{(k)}\right)-J^{\prime}(u), u^{(k)}-u\right\rangle \\
& +\sum_{n \in Z^{m}} \gamma_{n}\left(f_{n}\left(u_{n}^{(k)}\right)-f_{n}\left(u_{n}\right)\right)\left(u_{n}^{(k)}-u_{n}\right) \\
\leq & \left\langle J^{\prime}\left(u^{(k)}\right)-J^{\prime}(u), u^{(k)}-u\right\rangle \\
& +\sum_{n \in Z^{m}} \gamma_{n}\left(\epsilon\left(\left|u_{n}^{(k)}\right|+\left|u_{n}\right|\right)+c_{1}\left(\left|u_{n}^{(k)}\right|^{p-1}+\left|u_{n}\right|^{p-1}\right)\right) \\
& \times\left(u_{n}^{(k)}-u_{n}\right) \leq\left\langle J^{\prime}\left(u^{(k)}\right)-J^{\prime}(u), u^{(k)}-u\right\rangle \\
& +\bar{\gamma}^{1 / 2}\left(\epsilon\left(\left\|u^{(k)}\right\|_{l^{2}}+\|u\|_{l^{2}}\right)\right. \\
& \left.+c_{1}\left(\left\|u^{(k)}\right\|_{l^{2(p-1)}}^{p-1}+\|u\|_{l^{2(p-1)}}^{p-1}\right)\right) \\
\times & \times\left\|u^{(k)}-u\right\|_{l_{\gamma}^{2}}
\end{aligned}
$$

Therefore, combining (11), (24), (52), (53), (54), and the boundedness of $\left\{u^{(k)}\right\}$, it is clear that

$$
\lim _{k \rightarrow \infty}\left\|u^{(k)}-u\right\|_{l^{2}}=0
$$

and this means $J$ satisfies $(C)_{c}$ condition. The proof is complete.

Now, we are ready to prove Theorem 5.

Proof of Theorem 5. Let $a, \rho$, and $e \in l^{2}$ be obtained in Lemma 10.

Since $J(t e) \rightarrow-\infty$ as $|t| \rightarrow \infty$, there exists a real number $t_{0}$ such that

$$
\left\|t_{0} e\right\|_{l^{2}}>\rho, \quad J\left(t_{0} e\right)<0 .
$$

Immediately, we obtain

$$
\max \left\{J(0), J\left(t_{0} e\right)\right\}=0<a \leq \inf _{\|u\|=\rho} J(u) .
$$

Now that we have verified all assumptions of Lemma 4, we know $J$ possesses a $(C)_{c}$ sequence $\left\{u_{j}\right\} \subset l^{2}$ for the mountain pass level $c \geq a$ with

$$
c=\inf _{h \in \Gamma s \in[0,1]} \max _{(h)} J(h)
$$

where

$$
\Gamma=\left\{h \in C\left([0,1], l^{2}\right): h(0)=0, h(1)=t_{0} e\right\} .
$$

By Lemma 11, $\left\{u_{j}\right\}$ has a convergent subsequence $\left\{u_{j_{m}}\right\}$ such that $u_{j_{m}} \rightarrow u$ as $j_{m} \rightarrow+\infty$ for some bounded $u \in l^{2}$. Since $J \in C^{1}\left(l^{2}, R\right)$, we have

$$
\begin{aligned}
J\left(u_{j_{m}}\right) & \longrightarrow J(u), \\
\left(1+\left\|u_{j_{m}}\right\|_{l^{2}}\right) J^{\prime}\left(u_{j_{m}}\right) & \longrightarrow\left(1+\|u\|_{l^{2}}\right) J^{\prime}(u),
\end{aligned}
$$

as $j_{m} \rightarrow+\infty$. By the uniqueness of limit and the fact that $u$ is bounded, we obtain that $u$ is a nontrivial critical point of $J$ as the corresponding critical value $c \geq a>0$. Hence, (1) has at least one nontrivial solution $u$ in $l^{2}$.

Finally, we show that $u=\left\{u_{n}\right\}$ satisfies (28). In fact, similar to [39], for $n \in Z$, let

$$
w_{n}= \begin{cases}-\frac{\gamma_{n} f_{n}\left(u_{n}\right)}{u_{n}}, & u_{n} \neq 0, \\ 0, & u_{n}=0 ;\end{cases}
$$

then

$$
\bar{L} u_{n}=\omega u_{n}
$$

where

$$
\bar{L} u_{n}=L u_{n}+w_{n} u_{n}
$$

Clearly, $\lim _{|n| \rightarrow \infty} w_{n}=0$. Thus, the multiplication by $w_{n}$ is a compact operator in $l^{2}$, which implies that

$$
\sigma_{\text {ess }}(\bar{L})=\sigma_{\text {ess }}(L)
$$

where $\sigma_{\text {ess }}$ stands for the essential spectrum. Equation (62) means that $u=\left\{u_{n}\right\}$ is an eigenfunction that corresponds to the eigenvalue of finite multiplicity $\omega \notin \sigma_{\text {ess }}(\bar{L})$ of the operator $\bar{L}$. Equation (28) follows from the standard theorem on exponential decay for such eigenfunctions [1]. Now the proof of Theorem 5 is complete.

\section{Conflict of Interests}

The authors declare that there is no conflict of interests regarding the publication of this paper.

\section{Acknowledgments}

This work is supported by the Program for Changjiang Scholars and Innovative Research Team in University (no. IRT1226), the National Natural Science Foundation of China (no. 11171078), the Specialized Fund for the Doctoral Program of Higher Education of China (no. 20114410110002), and the Project for High Level Talents of Guangdong Higher Education Institutes.

\section{References}

[1] G. Teschl, Jacobi Operators and Completely Integrable Nonlinear Lattices, vol. 72, The American Mathematical Society, Providence, RI, USA, 2000.

[2] N. I. Karachalios, "A remark on the existence of breather solutions for the discrete nonlinear Schrodinger equation in infinite lattices: the case of site-dependent anharmonic parameters," Proceedings of the Edinburgh Mathematical Society II, vol. 49, no. 1, pp. 115-129, 2006.

[3] M. Willem, Minimax Theorems, vol. 24 of Progress in Nonlinear Differential Equations and Their Applications, Birkhäuser, Boston, Mass, USA, 1996. 
[4] W. Królikowski, B. Luther-Davies, and C. Denz, "Photorefractive solitons," IEEE Journal of Quantum Electronics, vol. 39, no. 1, pp. 3-12, 2003.

[5] G. Kopidakis, S. Aubry, and G. P. Tsironis, "Targeted energy transfer through discrete breathers in nonlinear systems," Physical Review Letters, vol. 87, no. 16, Article ID 165501, pp. 1-4, 2001.

[6] R. Livi, R. Franzosi, and G. Oppo, "Self-localization of BoseEinstein condensates in optical lattices via boundary dissipation," Physical Review Letters, vol. 97, no. 6, Article ID 060401, 2006.

[7] M. H. Jakubowski, K. Steiglitz, and R. Squier, "Information transfer between solitary waves in the saturable Schrödinger equation," Physical Review E, vol. 56, no. 6, pp. 7267-7272, 1997.

[8] A. Khare, K. Rasmussen, M. Samuelsen, and A. Saxena, "Exact solutions of the saturable discrete nonlinear Schrodinger equation," Journal of Physics A, vol. 38, no. 4, pp. 807-814, 2005.

[9] W. Królikowski and S. A. Holmstrom, "Fusion and birth of spatial solitons upon collision," Optics Letters, vol. 22, no. 6, pp. 369-371, 1997.

[10] J. W. Fleischer, T. Carmon, M. Segev, N. K. Efremidis, and D. N. Christodoulides, "Observation of discrete solitons in optically induced real time waveguide arrays," Physical Review Letters, vol. 90, no. 2, p. 023902/4, 2003.

[11] J. W. Fleischer, M. Segev, N. K. Efremidis, and D. N. Christodoulides, "Observation of two-dimensional discrete solitons in optically induced nonlinear photonic lattices," Nature, vol. 422, no. 6928, pp. 147-150, 2003.

[12] G. Arioli and F. Gazzola, "A remark on the existence of breather solutions for the discrete nonlinear Schrodinger equation in infinite lattices: the case of site-dependent anharmonic parameters," Proceedings of the Edinburgh Mathematical Society II, vol. 26, no. 6, pp. 1103-1114, 1996.

[13] S. Aubry, "Breathers in nonlinear lattices: existence, linear stability and quantization," Physica D: Nonlinear Phenomena, vol. 103, no. 1-4, pp. 201-250, 1997.

[14] S. Aubry, G. Kopidakis, and V. Kadelburg, "Variational proof for hard discrete breathers in some classes of Hamiltonian dynamical systems," Discrete and Continuous Dynamical Systems B, vol. 1, no. 3, pp. 271-298, 2001.

[15] J. Cuevas, P. G. Kevrekidis, and D. J. Frantzeskakis, "Discrete solitons in nonlinear Schrodinger lattices with a power-law nonlinearity," Physica D: Nonlinear Phenomena, vol. 238, no. 1, pp. 67-76, 2009.

[16] G. James, "Centre manifold reduction for quasilinear discrete systems," Journal of Nonlinear Science, vol. 13, no. 1, pp. 27-63, 2003.

[17] R. S. MacKay and S. Aubry, "Proof of existence of breathers for time-reversible or Hamiltonian networks of weakly coupled oscillators," Nonlinearity, vol. 7, no. 6, pp. 1623-1643, 1994.

[18] A. Pankov and V. Rothos, "Periodic and decaying solutions in discrete nonlinear Schrödinger with saturable nonlinearity," Proceedings of the Royal Society A: Mathematical, Physical and Engineering, vol. 464, no. 2100, pp. 3219-3236, 2008.

[19] S. Aubry, "Discrete breathers: localization and transfer of energy in discrete Hamiltonian nonlinear systems," Physica D. Nonlinear Phenomena, vol. 216, no. 1, pp. 1-30, 2006.

[20] S. Flach and A. V. Gorbach, "Discrete breathers-advances in theory and applications," Physics Reports, vol. 467, no. 1-3, pp. 1-116, 2008.

[21] S. Flach and C. R. Willis, "Discrete breathers," Physics Reports, vol. 295, no. 5, pp. 181-264, 1998.
[22] A. A. Sukhorukov and Y. S. Kivshar, "Generation and stability of discrete gap solitons," Optics Letters, vol. 28, no. 23, pp. 23452347, 2003.

[23] A. V. Gorbach and M. Johansson, "Gap and out-gap breathers in a binary modulated discrete nonlinear Schrödinger model," European Physical Journal D, vol. 29, no. 1, pp. 77-93, 2004.

[24] A. Mai and Z. Zhou, "Discrete solitons for periodic discrete nonlinear Schrodinger equations," Applied Mathematics and Computation, vol. 222, pp. 34-41, 2013.

[25] G. Sun, "On standing wave solutions for discrete nonlinear Schrödinger equations," Abstract and Applied Analysis, vol. 2013, Article ID 436919, 6 pages, 2013.

[26] D. Ma and Z. Zhou, "Existence and multiplicity results of homoclinic solutions for the DNLS equations with unbounded potentials," Abstract and Applied Analysis, vol. 2012, Article ID 703596, 15 pages, 2012.

[27] X. Tang and J. Chen, "Infinitely many homoclinic orbits for a class of discrete Hamiltonian systems," Advances in Difference Equations, vol. 2013, article 242, 2013.

[28] X. H. Tang and X. Lin, "Infinitely many homoclinic orbits for discrete Hamiltonian systems with subquadratic potential," Journal of Difference Equations and Applications, vol. 19, no. 5, pp. 796-813, 2013.

[29] X. H. Tang and X. Y. Lin, "Homoclinic solutions for a class of second order discrete Hamiltonian systems," Acta Mathematica Sinica, vol. 28, no. 3, pp. 609-622, 2012.

[30] Z. Zhou and J. Yu, "On the existence of homoclinic solutions of a class of discrete nonlinear periodic systems," Journal of Differential Equations, vol. 249, no. 5, pp. 1199-1212, 2010.

[31] J. Kuang and Z. Guo, "Homoclinic solutions of a class of periodic difference equations with asymptotically linear nonlinearities," Nonlinear Analysis, vol. 89, pp. 208-218, 2013.

[32] G. Chen and S. Ma, "Discrete nonlinear Schrödinger equations with superlinear nonlinearities," Applied Mathematics and Computation, vol. 218, no. 9, pp. 5496-5507, 2012.

[33] G. Chen and S. Ma, "Ground state and geometrically distinct solitons of discrete nonlinear Schrödinger equations with saturable nonlinearities," Studies in Applied Mathematics, vol. 131, no. 4, pp. 389-413, 2013.

[34] A. Mai and Z. Zhou, "Ground state solutions for the periodic discrete nonlinear Schrödinger equations with superlinear nonlinearities," Abstract and Applied Analysis, vol. 2013, Article ID 317139, 11 pages, 2013.

[35] S. Ma and Z. Wang, "Multibump solutions for discrete periodic nonlinear Schrödinger equations," Journal of Applied Mathematics and Physics, vol. 64, no. 5, pp. 1413-1442, 2013.

[36] Z. Zhou and J. S. Yu, "Homoclinic solutions in periodic nonlinear difference equations with superlinear nonlinearity," Acta Mathematica Sinica, vol. 29, no. 9, pp. 1809-1822, 2013.

[37] C. A. Stuart, "Locating Cerami sequences in a mountain pass geometry," Communications in Applied Analysis, vol. 15, no. 24, pp. 569-588, 2011.

[38] S. B. Liu and S. J. Li, "Infinitely many solutions for a superlinear elliptic equation," Acta Mathematica Sinica, vol. 46, no. 4, pp. 625-630, 2003.

[39] A. Pankov, "Gap solitons in periodic discrete nonlinear Schrödinger equations," Nonlinearity, vol. 19, no. 1, pp. 27-40, 2006. 


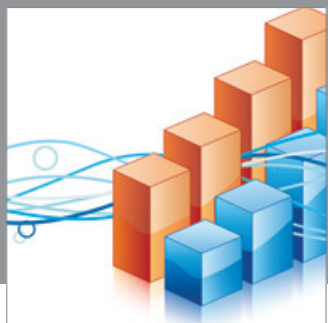

Advances in

Operations Research

mansans

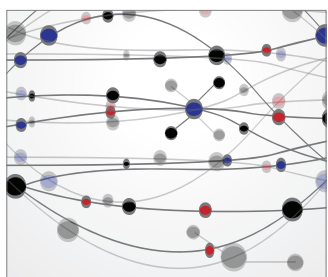

The Scientific World Journal
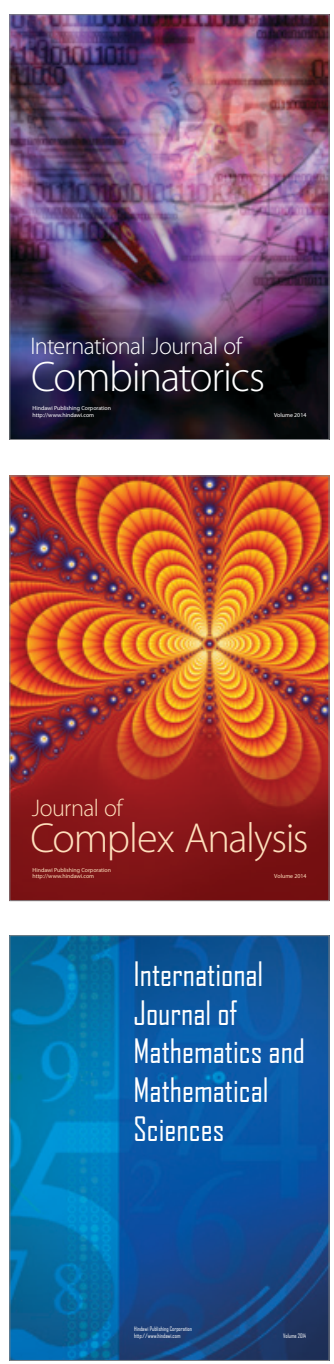
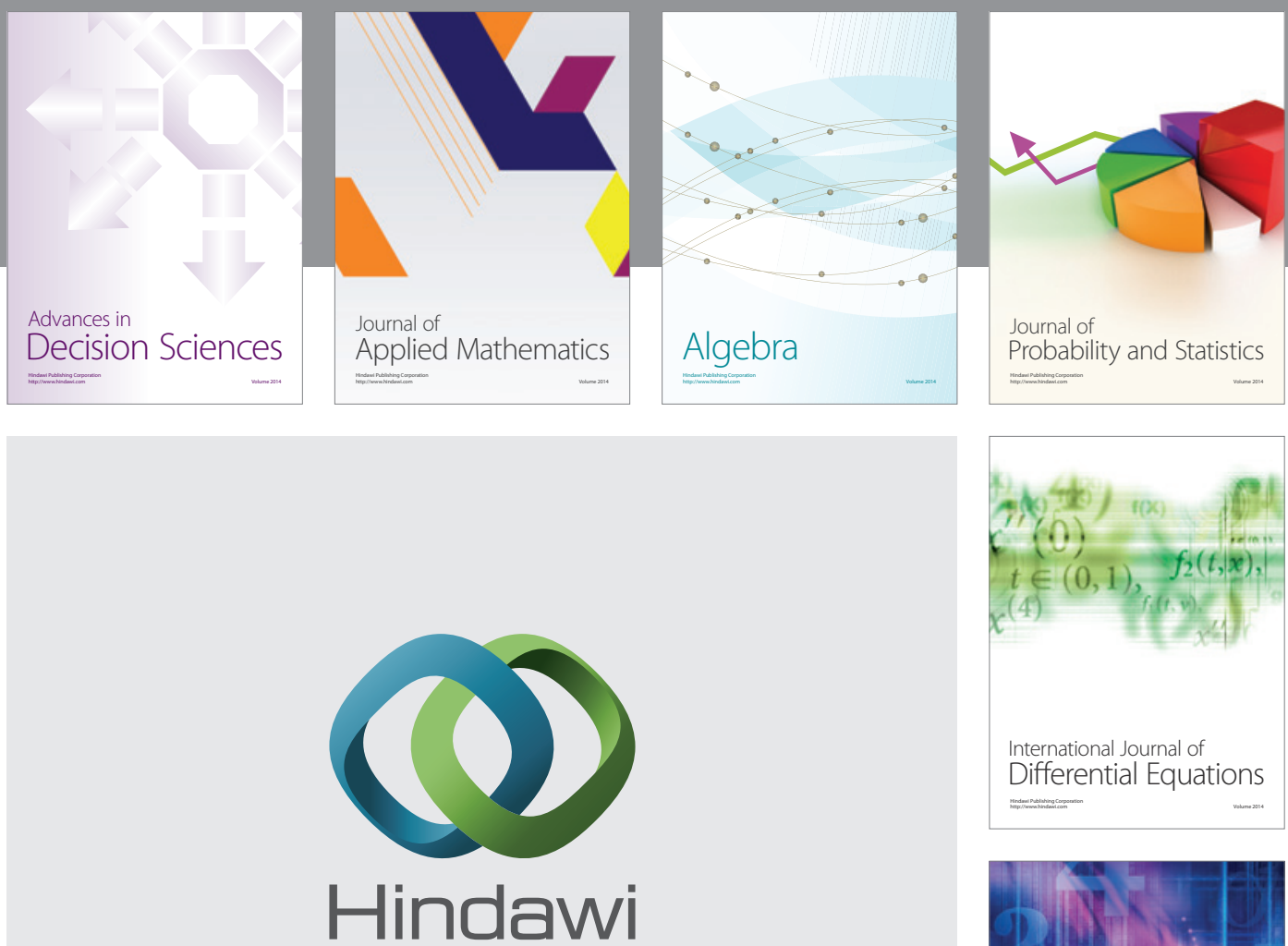

Submit your manuscripts at http://www.hindawi.com
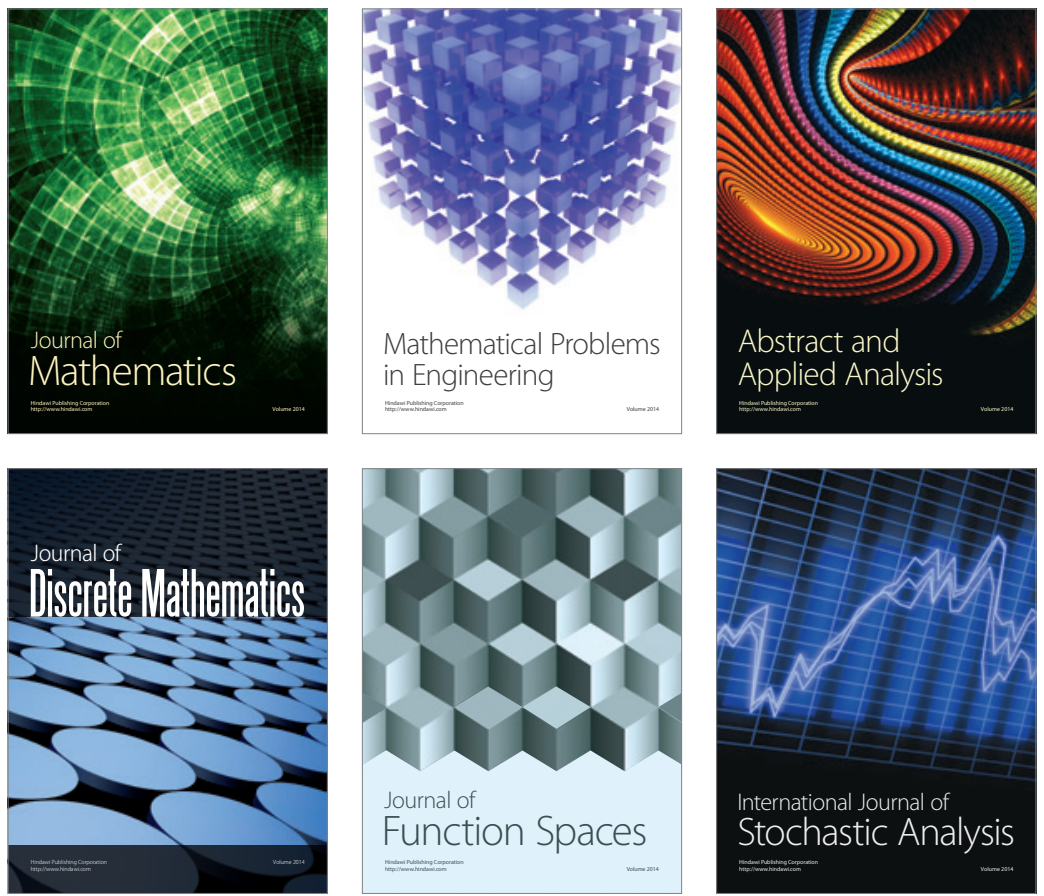

Journal of

Function Spaces

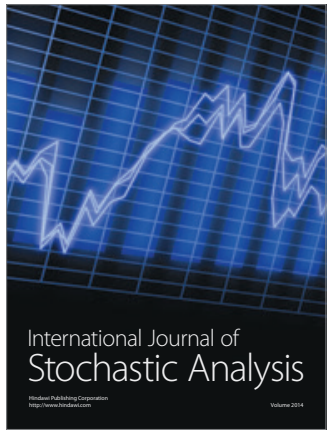

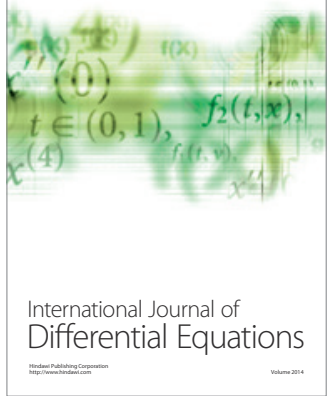
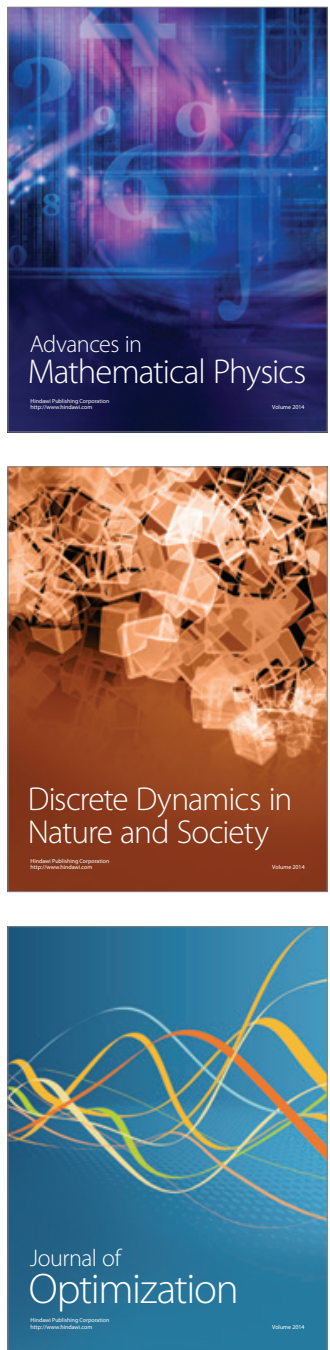